\title{
Impacto de la reconstrucción mamaria sobre la autoestima y la imagen corporal en pacientes con cáncer de mama*
}

\author{
The impact of breast reconstruction on self-esteem \\ and body image in patients with breast cancer
}

Recibido: septiembre 29 de 2009 | Revisado: julio 10 de 2010 | Aceptado: octubre 20 de 2010

\author{
MARÍA EsTHer Rincón FERNÁNDEZ ** \\ María Ángeles Pérez SAN Gregorio**** \\ MERCEDES BORdA MÁs \\ Agustín MARTÍN RODRÍGUEZ **** \\ Facultad de Psicología, Universidad de Sevilla, España
}

SICI: 2011-2777(201203)11:1<25:IRMAIC>2.0.CO;2-X

Para citar este artículo: Rincón, M. E., Pérez, M. A., Borda, M. \& Martín, A. (2012). Impacto de la reconstrucción mamaria sobre la autoestima y la imagen corporal en pacientes con cáncer de mama. Universitas Psychologica, 11(1), 25-41.

* Artículo de investigación original en Psicología de la Salud.

** Facultad de Psicología. Departamento de Persona-lidad, Evaluación y Tratamiento Psicológicos. C/. Camilo José Cela, s/n. 41018, Sevilla (España). Tel: 954556939.E-mail: erincon@us.es.

*** E-mails: anperez@us.es, mborda@us.es.

*****E-mail: amartinr@us.es. Researcher-ID: H-52672011.

\section{RESUMEN}

El tratamiento quirúrgico del cáncer de mama puede alterar el aspecto físico de las pacientes y producirles importantes secuelas psicológicas. Para comprobar si el nivel de satisfacción con el resultado estético, así como la autoestima o la imagen corporal de estas pacientes difería según el tipo de cirugía realizada (mastectomía unilateral o reconstrucción mamaria), se estudiaron un total de 72 pacientes. Para ello, se emplearon una Encuesta Psicosocial, la Escala de Autoestima de Rosenberg (EAR), el Cuestionario de Calidad de Vida de la EORTC (QLQ-BR23) y el Test de Medición del Grado de Satisfacción Corporal. En general, las pacientes reconstruidas mostraron una mayor satisfacción estética, así como una mayor autoestima y un menor deterioro de su imagen corporal.

Palabras clave autores:

Cáncer de mama, calidad de vida, satisfacción estética, autoestima, imagen corporal. Palabras clave descriptores:

Mastectomía, reconstrucción mamaria, psico-oncología.

\section{A B S T R A C T}

The surgical treatment of breast cancer can change patients' physical appearance and lead to important physical sequelae. Therefore, in order to ascertain whether the level of satisfaction with the aesthetic outcome, as well as with regard to self-esteem or the body image in these patients, differs depending on the kind of surgery undergone (unilateral mastectomy or breast reconstruction), a total of 72 patients were studied. To this end, a Psychosocial Survey, the Rosenberg Self-Esteem (RSE) Scale, the EORTC Quality of Life Questionnaire (QLQ-BR23), and the Test for the Measurement of Body Satisfaction were used. In general, patients who underwent reconstruction showed a higher degree of aesthetic satisfaction, as well as greater self-esteem and a lower degree of dissatisfaction regarding their body image.

Key words authors:

Breast cancer, quality of life, aesthetic satisfaction, self-esteem, body image.

Key words plus:

Mastectomy, breast reconstruction, psycho-oncology. 


\section{Introducción}

Con frecuencia, el cáncer y sus tratamientos pueden alterar el aspecto físico de las personas que lo padecen. Estos cambios pueden ser temporales, como la caída del cabello, o permanentes, como los efectos provocados por la cirugía (Fernández, 2004). En las pacientes con cáncer de mama se ha observado que estos cambios están asociados con la insatisfacción corporal y con el deterioro de la imagen corporal, además de ir acompañados de un gran impacto emocional (Avis, Crawford \& Manuel, 2004; Hopwood, Fletcher, Lee \& Al Ghazal, 2001; Vázquez, López \& Martín, 2004; White, 2000). Así, autores como Sellick y Edwardson (2007) hallaron que el $22.6 \%$ de las 773 pacientes recién diagnosticadas con cáncer de mama, presentaban niveles patológicos de ansiedad, junto a un $5.3 \%$ que manifestaron niveles patológicos de depresión.

Debido a esto, la investigación ha tratado de determinar cuáles son aquellas variables que permiten predecir un mayor grado de bienestar emocional en estas pacientes. En este sentido, autores como Ganz (2008) han mostrado que los principales factores de riesgo para un mayor malestar emocional de estas mujeres eran los siguientes: edad, trastorno mental previo o historia de malestar emocional anterior, presencia de otras enfermedades comórbidas y escaso apoyo social. De este modo, aquellas pacientes de entre 30 y 40 años con historia previa de trastorno depresivo, dolor, limitaciones físicas o alguna otra enfermedad concurrente y que contasen además con escaso apoyo social e instrumental, constituirían un perfil de riesgo con gran probabilidad de padecer un mayor deterioro emocional. Otros factores que se han estudiado para comprender el ajuste psicológico de la paciente a su enfermedad han sido el grado de "percepción de control" (Neipp, López-Roig, Terol \& Pastor, 2009) o las estrategias de afrontamiento empleadas por las pacientes para luchar contra el cáncer (Anarte, Esteve, Ramírez, López \& Amorós, 2001; Cardenal, Ortiz-Tallo, Martín-Frias \& Martínez-Lozano, 2008; Collie et al., 2005; Font \& Cardoso, 2009). En relación con los tratamientos médicos, la mayoría de los estudios coinciden en afirmar la ausencia de dife- rencias significativas respeto al malestar psicológico padecido por las pacientes sometidas a diferentes tratamientos médicos (Mose et al., 2001; Stiegelis, Ranchor \& Sanderman, 2004; Whelan, Levine, Julian, Kirkbride \& Skingley, 2000). Sin embargo, otros autores señalan que aquellas mujeres tratadas únicamente con quimioterapia, muestran mayores niveles de malestar emocional (Buick et al., 2000).

No cabe duda de que los cambios debidos al cáncer y sus tratamientos influyen negativamente en la imagen que las pacientes tienen de su propio cuerpo (White, 2000), provocando desórdenes afectivos, así como cambios en la sexualidad y en la autoestima (Hopwood \& Maguire, 1998). De todos los diagnósticos oncológicos, el cáncer de mama ha sido destacado por su mayor efecto en la imagen corporal de las pacientes (Ferrell, Grant, Funk, Otis-Green \& García, 1997). Sin embargo, la mayor relevancia no está representada por el cambio objetivo que ha sufrido la mujer, sino por el significado que le atribuye (Raich, 2000).

Debido a esto, los investigadores han intentado delimitar cuáles son las intervenciones quirúrgicas que consiguen un mayor grado de satisfacción en las pacientes. Numerosas investigaciones han afirmado que las pacientes reconstruidas muestran un elevado nivel de satisfacción con el resultado de la intervención (Alderman, Wilkins, Lowery, Kim \& Davis, 2000; Contant, Van Wersch, Wiggers, Tjong \& Van Geel, 2000; Elder et al., 2005), siendo mayor que el manifestado por las mujeres mastectomizadas (Janni et al., 2001; Nicholson, Leinster $\&$ Sassoon, 2007).

En relación con la influencia de la personalidad en la adaptación de las pacientes con cáncer de mama, se han estudiado aspectos tales como el patrón de conducta Tipo C (Torres, 2006) o el nivel de neuroticismo. Así, se ha afirmado que una mayor puntuación en neuroticismo se relaciona con un menor nivel de autoestima (Buunk, Collins, Taylor, VanYperen \& Dakof, 1990).

Cabe destacar que existen diversos factores que median entre el impacto del cáncer de mama y de sus tratamientos, sobre el nivel de autoestima de las pacientes. La mayoría de estos factores se basan en la edad de la paciente, en su funcionamiento 
premórbido, en el estadio de evolución de la enfermedad, así como en los tipos de tratamientos oncológicos que le sean administrados (Cordova, Cunninggham, Carlson \& Andrykowski, 2001). Por otro lado, ni el nivel educativo ni el estado civil ni el tipo de cirugía mamaria, se han relacionado con la autoestima manifestada por las pacientes, hallándose tan solo una relación positiva entre el nivel de autoestima y la percepción general acerca de su calidad de vida (Manos, Sebastián, Bueno, Mateos \& De la Torre, 2005).

Con respecto al tratamiento quirúrgico del cáncer de mama, la investigación ha sugerido resultados contradictorios sobre el ajuste psicológico de las pacientes. En este sentido, la mastectomía ha sido señalada como aquella opción terapéutica que genera un mayor deterioro en la imagen corporal y en el nivel de funcionamiento sexual de las pacientes, siendo esta elección quirúrgica, aplicada junto a la quimioterapia adyuvante la que provoca un mayor deterioro en la calidad de vida (Muñoz, 2010). Es por esto que la reconstrucción mamaria representa un procedimiento quirúrgico para revertir la deformidad causada por la mastectomía, siendo su principal objetivo la restitución de la imagen corporal y del bienestar psicológico de las pacientes postmastectomía (Marín-Gutzke \& Sánchez-Olaso, 2010).

De este modo, se ha señalado que las mujeres sometidas a una reconstrucción mamaria manifestaban un mayor nivel de autoestima y un menor deterioro en la imagen corporal que las mujeres mastectomizadas sin reconstrucción (Al-Ghazal, Fallowfield \& Blamey, 2000). Estas mejorías se han comprobado incluso a lo largo del tiempo, hallando incrementos significativos en la imagen corporal y en el bienestar emocional de las pacientes (Arora et al., 2001), manteniendo un buen nivel de calidad de vida, de adaptación social y de funcionamiento sexual, transcurrido un año (Rubino, Figus, Lorettu \& Sechi, 2007), e incluso dos años después de la reconstrucción mamaria (Atisha et al., 2008).

Contrario a lo anteriormente expuesto, diversos autores han afirmado que aspectos tales como la calidad de vida (Nicholson et al., 2007; Nissen et al., 2001), la imagen corporal (Nissen et al., 2001) o el nivel de ansiedad y depresión (Nicholson et al., 2007), no se han diferenciado en mujeres con cirugía conservadora, con reconstrucción tras mastectomía o en aquellas pacientes mastectomizadas no reconstruidas. Más aún, las pacientes sometidas a reconstrucción mamaria mostraban mayores alteraciones del estado de ánimo junto a un menor sentimiento de bienestar (Nissen et al., 2001). En este sentido, otros investigadores (Millar, Purushotham, McLatchie, George \& Murray, 2005) compararon pacientes sometidas a cirugía conservadora con pacientes mastectomizadas, sin determinar diferencias significativas en el nivel de malestar psicológico en ambos grupos. Igualmente, Harcourt et al. (2003) tampoco hallaron discrepancias con respecto a la calidad de vida ni a la imagen corporal, al contrastar pacientes mastectomizadas con reconstrucción inmediata o con reconstrucción diferida. Sin embargo, Min et al. (2010) manifestaron recientemente que las pacientes con reconstrucción mamaria inmediata experimentaban un menor deterioro de la imagen corporal y una menor preocupación por el futuro que las pacientes sometidas a cirugía conservadora.

En relación con el tiempo transcurrido desde el diagnóstico de cáncer, algunos estudios han obtenido correlaciones positivas entre este y un mayor bienestar psicológico percibido por las pacientes (Cordova et al., 2001), mientras que en otros, las correlaciones no son significativas (Lechner, Zakowski, Antoni, Greenhawt \& Block, 2003; Manne et al., 2004). Así, autores como Quintard y Lakdja (2008) hallaron que las pacientes con cáncer de mama, sometidas a mastectomía y a tumorectomía (extirpación exclusiva del tumor y márgenes quirúrgicos adyacentes), incrementaban progresivamente su nivel de sintomatología depresiva con el paso del tiempo, mientras que la sintomatología ansiosa decrecía. Otros autores como Costa y Gil (2008) determinaron que durante el primer año del diagnóstico, las respuestas cognitivas ante el diagnóstico no se modificaban significativamente.

Según esto, cabe mencionar que los resultados obtenidos en relación con el ajuste psicológico de las pacientes con cáncer de mama en función del tratamiento quirúrgico llevado a cabo, ofrecen 
perspectivas contrapuestas, las cuales incluyen la interrelación de distintas variables que podrían mediar en el nivel de autoestima y de la calidad de vida experimentado por estas mujeres.

De este modo, el presente estudio contempla los siguientes objetivos: a) en primer lugar, comprobar si las pacientes con cáncer de mama se diferencian en una serie de variables psicológicas (satisfacción con el resultado estético de la intervención quirúrgica, grado de neuroticismo, autoestima, calidad de vida y grado de satisfacción corporal), según el tipo de cirugía mamaria que se les haya practicado: mastectomía unilateral (simple o radical modificada) o reconstrucción mamaria postmastectomía (inmediata o diferida); b) en segundo lugar, detectar si estas mismas variables psicológicas se encuentran relacionadas entre sí y c) en tercer lugar, determinar igualmente si las variables anteriormente mencionadas, se modifican en función del tiempo transcurrido (meses) desde la comunicación del diagnóstico oncológico y desde la intervención quirúrgica.

\section{Material y métodos}

\section{Participantes}

Para llevar a cabo este estudio, se constituyeron dos grupos: un primer grupo formado por aquellas mujeres que habían sido intervenidas mediante mastectomía sin reconstrucción posterior (Grupo 1) y un segundo grupo de pacientes que sí habían sido sometidas a cirugía reconstructiva mamaria postmastectomía (Grupo 2).

Estos dos grupos se subdividieron a su vez en cuatro subgrupos equivalentes: pacientes con mastectomía simple unilateral sin reconstrucción mamaria (subgrupo $1 ; N=18$ ), pacientes con mastectomía radical modificada unilateral sin reconstrucción mamaria (subgrupo $2 ; N=18$ ), pacientes con reconstrucción mamaria inmediata (subgrupo 3; $N=18$ ) y pacientes con reconstrucción mamaria diferida (subgrupo 4; $N=18$ ). Todas estas intervenciones quirúrgicas se habían realizado en el Hospital Universitario Virgen Macarena de Sevilla, durante los años 2005, 2006 y 2007.
A continuación se describen las principales características sociodemográficas y clínicas de ambos grupos:

- El Grupo 1 estaba constituido por 36 mujeres a quienes se les había practicado una mastectomía unilateral (simple o radical modificada) por cáncer de mama, siendo la edad media de estas pacientes de 51.39 años (DE 10.46, rango 30-66 años). El tiempo medio transcurrido desde que se les había practicado la intervención quirúrgica (mastectomía) fue de 17.39 meses (DE 9.88, rango 2.6-33.89 meses), mientras que el tiempo medio acontecido desde que se les comunicó el diagnóstico de cáncer osciló entre los 4.27 y los 35.47 meses como máximo (M 19.07 y DE 9.89 meses). La mayoría de estas mujeres estaban casadas o tenían pareja estable (83.3 \%), siendo amas de casa (44.4\%) con un nivel de estudios primarios (66.7\%). Casi la totalidad de las pacientes habían sido diagnosticadas de un carcinoma ductal infiltrante (91.7\%), con estadios predominantes de IIA (44.4\%) y de IIB (27.8\%), tratadas de manera adyuvante con quimioterapia administrada junto a radioterapia (33.3 \%) o junto a hormonoterapia (22.2\%). El 88.9 \% había sido sometido a linfadenectomía axilar. Los síntomas secundarios referidos en su mayoría fueron debilidad (88.9\%), náuseas (86.1\%), alopecia (86.1\%) y aumento de peso (72.2 \%).

- El Grupo 2 estaba constituido por 36 mujeres que habían sido sometidas a reconstrucción mamaria (inmediata o diferida) tras la mastectomía por cáncer de mama, con una edad media de 47.75 años (DE 6.59, rango 32-60 años). El tiempo medio transcurrido desde que se les practicó la reconstrucción mamaria fue de 12.8 meses (DE 7.39, rango 3.47-29.2 meses), habiendo sido diagnosticadas desde hacía 45.74 meses como promedio (DE 28.1, rango 6.83-138.47 meses). La mayoría de estas mujeres estaban casadas o tenían pareja estable $(83.3 \%)$ y desarrollaban su actividad laboral como amas de casa (36.1\%), con un nivel de estudios secundario (36.1\%). Más de la mitad de estas pacientes habían recibido un diagnóstico de carcinoma ductal infiltrante (69.4\%) en estadio IIA (55.6\%), por lo que se les prescribió un tratamiento de hormonoterapia (25\%) o bien de 
quimioterapia junto a hormonoterapia (22.2\%). Los síntomas secundarios más frecuentes a estos tratamientos fueron alopecia (69.4\%) y aumento de peso $(69.4 \%)$, siendo sometidas a linfadenectomía axilar un $69.4 \%$ de ellas.

\section{Instrumentos}

\section{Encuesta psicosocial}

Elaborada por los autores para la presente investigación y constituida por 30 ítems que evaluaban datos sociodemográficos (edad, estado civil, nivel de estudios, profesión, situación laboral, etc.) y médicos (tipo de cáncer de mama, estadio, fecha del diagnóstico, tipo de cirugía realizada, tratamientos médicos recibidos, etc.) (ítems 1 a 25). Asimismo, se incluyó un ítem en el que las pacientes manifestaban su grado de satisfacción con el resultado estético de la intervención quirúrgica (ítem 26), conformado por una escala tipo Likert de tres opciones $(1=$ poco satisfecha, 2 = medianamente satisfecha, $3=$ muy satisfecha). Además se añadieron otros cuatro ítems que constituyesen indicadores del grado de neuroticismo expresado por las pacientes, es decir, del nivel en el que las mujeres con cáncer de mama manifestaban un patrón conductual caracterizado por la inestabilidad emocional. Estos enunciados fueron extraídos del cuestionario de Personalidad de Eysenck, EPQR-A (Francis, Brown \& Philipehalk, 1992), correspondiendo a cuatro de los seis ítems que miden dicha dimensión. En concreto, se incluyeron los ítems número dos, diez, catorce y veintidós (ítems 27 al 30), siendo extraídos de la versión española de este cuestionario (Sandín, Valiente, Chorot, Olmedo \& Santed, 2002). En el artículo original, la escala de Neuroticismo mantiene un coeficiente alpha entre 0.70 y 0.77 , lo que es muy elevado en escalas de tan solo seis ítems. En cuanto a la validez concurrente, ha sido obtenida al comparar las escalas del EPQR-A con la versión anterior, EPQR, obteniendo índices de 0.92 y 0.94 en la escala de Neuroticismo (Francis et al., 1992). El alfa de Cronbach de las diferentes subescalas de la versión española osciló entre 0.74 y 0.78 (Sandín, Valiente, Olmedo, Chorot \& Santed, 2002).

\section{Escala de Autoestima ([EAR], Rosenberg, 1965)}

Constituida por diez ítems referidos a la valoración global que hace el individuo respecto de sí mismo. El rango de la escala oscila entre diez y cuarenta, siendo su sistema de respuesta una escala tipo Likert con cuatro opciones $(1=$ muy de acuerdo, $2=$ de acuerdo, 3 = en desacuerdo y $4=$ totalmente en desacuerdo). En su forma original, la escala presenta un nivel de fiabilidad test-retest que oscila entre 0.82 y 0.88 , así como un nivel de consistencia interna medida mediante el coeficiente alpha de Cronbach que va desde 0.77 hasta 0.88 (Rosenberg, 1986), demostrándose igualmente su unidimensionalidad. En estudios realizados con población española, los datos muestran un coeficiente alfa de Cronbach de 0.87 y un índice de fiabilidad test-retest que oscila entre 0.72 y 0.74 (Vázquez, Jiménez \& Vázquez, 2004). En estudios realizados con pacientes oncológicos, su validez queda demostrada con valores de consistencia interna entre 0.76 y 0.87 (Curbow \& Somerfield, 1991).

\section{Cuestionario de Calidad de Vida para Cáncer de Mama ([EORTC QLQ- BR23], Sprangers et al., 1996)}

Este cuestionario incluye 23 ítems que valoran áreas relacionadas con las diferentes modalidades de tratamiento (cirugía, radioterapia, quimioterapia, hormonoterapia) y otros aspectos de la calidad de vida más específicamente afectados en el cáncer de mama, como la imagen corporal o la sexualidad (Arraras et al., 2001). Todas estas escalas e ítems individuales emplean un formato de respuesta tipo Likert de cuatro puntos, las cuales se transforman en una puntuación de 0 a $100(1=$ en absoluto, $2=$ un poco, 3 = bastante y $4=$ mucho). En el artículo original, Sprangers et al. (1996) validaron el cuestionario con una muestra de pacientes con cáncer de mama de diferentes países tales como Alemania, España y América. El coeficiente alfa de Cronbach obtenido fue más bajo en las pacientes españolas (rango de 0.46 a 0.94), siendo el más elevado el hallado en las mujeres americanas (rango de $0.7 \mathrm{a}$ 
0.91). Este instrumento ha sido validado en población española por Arraras et al. (2001).

Test de Medición del Grado de

Satisfacción Corporal (Raich, Torras

ES Sánchez-Carracedo, 2001)

Diseñado por García-Tornel y Gaspá (1997) y adaptado por Raich et al. (2001), compuesto por 44 ítems, constituye un medio con base en el que la paciente puede autoevaluar las diferentes partes de su cuerpo y su aspecto físico general, según un dibujo del cuerpo humano. Así, la paciente califica cada parte de su cuerpo de 0 a 10, indicando si alguna de dichas zonas corporales representa un problema estético para ella. En los estudios de fiabilidad del cuestionario (Miró, 2006), se ha obtenido un coeficiente alfa de Cronbach de 0.88 , para las puntuaciones que valoran cada parte del cuerpo, así como un 0.81 para el resto del cuestionario. En relación con la validez del instrumento, se ha obtenido una estructura bifactorial, al analizar las puntuaciones en cada una de las distintas partes del cuerpo.

\section{Procedimiento}

Los criterios de inclusión que se tuvieron en cuenta para seleccionar a las participantes en este estudio fueron: ser mujer, con edad comprendida entre los 25 y los 66 años, tener un diagnóstico de cáncer de mama en estadio I, II o III, encontrarse en un intervalo libre de enfermedad sin que tras la intervención se hubiera diagnosticado una recidiva o metástasis del cáncer, no tener una historia de patología neoplásica anterior, no haber sido intervenida mediante mastectomía o reconstrucción mamaria por cáncer de mama con anterioridad, tener un nivel de lectura y escritura que posibilitara la cumplimentación de las distintas escalas empleadas, no haber estado nunca en tratamiento psiquiátrico o psicológico, no estar en el momento de la entrevista bajo medicación psicoactiva y no presentar en el momento de la evaluación ninguna patología grave o incapacitante. Todas las pacientes reconstruidas debían haber completado el proceso quirúrgico de la reconstrucción mamaria, a excepción del complejo areola-pezón.

La selección de las pacientes se realizó en función de la base de datos del Hospital Universitario Virgen Macarena de Sevilla, en la que constaban todas las intervenciones quirúrgicas realizadas, mediante un método de muestro por cuotas (Salkind, 1999). Del total de las intervenciones, se seleccionaron 150 mujeres, según los criterios de inclusión de este estudio. De ellas, solo se consiguió establecer contacto telefónico con 110 pacientes de las cuales 6 se negaron a participar en el estudio, siendo por tanto 104 el total de mujeres que se citaron para acudir a la evaluación psicológica. Se encontró que 20 de ellas no acudieron finalmente a la evaluación. Por ello, el total de las evaluaciones realizadas fue de 84 , de las cuales se excluyeron un total de 12 pacientes (7 por incumplir los criterios de inclusión en el estudio ya que 5 estaban en tratamiento psiquiátrico y/o psicológico y 2 presentaban historia anterior de cáncer; y 5 por formar parte del estudio piloto previo). Así pues, se incluyeron como válidas un total de 72 evaluaciones.

Todas las participantes del estudio fueron evaluadas por una misma investigadora en una misma sala. Previamente, se informaba a las pacientes de los objetivos y metodología de la investigación, mediante la Hoja de Información y se obtenía el consentimiento informado por escrito. La Comisión de Ética e Investigación Sanitaria del Hospital colaborador, aprobó la idoneidad y puesta en marcha del presente estudio.

\section{Análisis de datos}

Los datos obtenidos fueron analizados con el paquete estadístico SPSS (Versión 14.0). Los estadísticos utilizados para las comparaciones intergrupales eran escogidos en función de la unidad de medida de las variables consideradas: nominales (chi-cuadrado), ordinales (U de Mann-Whitney) o cuantitativas ( $t$ de Student-Fisher). Asimismo, se empleó el coeficiente de correlación de Pearson para determinar el grado de asociación lineal entre dos variables de carácter cuantitativo. 


\section{Resultados}

Atendiendo al primer objetivo planteado en esta investigación, aplicamos la prueba de normalidad (prueba de Kolmogorov-Smirnov) en las variables evaluadas: satisfacción con el resultado estético de la intervención quirúrgica, neuroticismo, autoestima, calidad de vida y grado de satisfacción corporal. Posteriormente, se empleó la prueba $T$ para igualdad de medias, en aquellas variables que seguían una distribución normal, así como la prueba de $U$ de Mann-Whitney, en aquellas variables que no se ajustaban a la normalidad en su distribución. Las variables de naturaleza nominal se analizaron con la prueba chi-cuadrado de Pearson, siempre que cumpliesen los requisitos para poder aplicarla.

Las pacientes reconstruidas presentaron una satisfacción significativamente mayor con el resultado estético de la intervención quirúrgica $(p<0.05)$, frente a las mujeres mastectomizadas. Sin embargo, el nivel de neuroticismo fue equivalente entre ambos grupos de pacientes $(p>0.05)$. Por el contrario, las pacientes con reconstrucción mamaria mostraron un nivel de autoestima significativamente mayor $(p<0.01)$ que las mujeres mastectomizadas. Igualmente, las mujeres reconstruidas manifestaron una mejor calidad de vida, expresada mediante un menor deterioro de la imagen corporal $(p<0.01)$, junto a una menor preocupación por el futuro $(p<0.01)$ o por la pérdida del cabello $(p<0.01)$. Asimismo, describieron una menor sintomatología experimentada en el brazo debido a la cirugía $(p<0.01)$, en la mama provocada por la radioterapia $(p<0.01)$, o secundaria a la quimioterapia $(p<0.05)$ y a la hormonoterapia $(p<0.01)$. No se determinaron diferencias significativas entre grupos en relación con el funcionamiento sexual o al disfrute del sexo $(p>0.05)$ (Tabla 1).

TABLA 1

Comparación entre pacientes mastectomizadas y reconstruidas en satisfacción con el resultado estético de la intervención mamaria, neuroticismo, autoestima y calidad de vida

\begin{tabular}{|c|c|c|c|c|}
\hline \multirow[t]{2}{*}{ Variables } & \multicolumn{2}{|c|}{ Tipo de cirugía } & \multirow{2}{*}{$\begin{array}{l}\text { Estadístico de } \\
\text { contraste }\end{array}$} & \multirow[t]{2}{*}{$p$} \\
\hline & $\begin{array}{c}\text { Mastectomía } \\
(N=36) \\
M(D E)\end{array}$ & $\begin{array}{c}\text { Reconstrucción } \\
(\mathrm{N}=36) \\
M(D E)\end{array}$ & & \\
\hline \multicolumn{5}{|l|}{$\begin{array}{l}\text { Satisfacción resultado estético } \\
\text {. }\end{array}$} \\
\hline Puntuación total satisfacción & $2.97(0.16)$ & $2.81(0.46)$ & $\mathrm{U}=557.5$ & $0.047^{*}$ \\
\hline \multicolumn{5}{|l|}{ Neuroticismo } \\
\hline Puntuación total neuroticismo & $2.33(1.74)$ & $2.36(1.64)$ & $\mathrm{U}=645.5$ & 0.976 \\
\hline \multicolumn{5}{|l|}{ Autoestima } \\
\hline Puntuación total autoestima & $30.33(5.09)$ & $34.06(5.27)$ & $t(70)=-3.07$ & $0.003 * *$ \\
\hline \multicolumn{5}{|l|}{ Calidad de vida } \\
\hline Imagen Corporal $^{\circ}$ & $3.04(0.72)$ & $2.02(1.04)$ & $\mathrm{t}(62.48)=4.81$ & $0.000 * *$ \\
\hline Funcionamiento Sexual a & $1.69(0.71)$ & $1.84(0.83)$ & $\mathrm{U}=590$ & 0.493 \\
\hline Disfrute del Sexo ${ }^{a}$ & $1.5(0.73)$ & $1.75(0.84)$ & $\mathrm{U}=544.5$ & 0.191 \\
\hline Preocupación por el futuro ${ }^{\circ}$ & $3(1.01)$ & $2.11(1)$ & $\mathrm{U}=350$ & $0.001 * *$ \\
\hline Síntomas del brazo por la cirugía ${ }^{\circ}$ & $2.67(1)$ & $1.99(0.83)$ & $\mathrm{t}(70)=3.1$ & $0.003 * *$ \\
\hline Síntomas de la mama por la Radioterapia ${ }^{\circ}$ & $2.49(0.96)$ & $1.79(0.74)$ & $t(65.82)=3.4$ & $0.001 * *$ \\
\hline Síntomas secundarios por la Quimioterapia ${ }^{\circ}$ & $1.29(0.51)$ & $1.06(0.25)$ & $\mathrm{U}=487.5$ & $0.011 *$ \\
\hline Síntomas secundarios por la Hormonoterapia ${ }^{\circ}$ & $2.71(0.58)$ & $1.74(0.71)$ & $t(70)=6.32$ & $0.000 * *$ \\
\hline Preocupación por la caída del cabello ${ }^{\circ}$ & $1.52(0.84)$ & $1.06(0.33)$ & $\mathrm{U}=464.5$ & $0.003 * *$ \\
\hline
\end{tabular}

Nota. $N=72 .{ }^{a}$ A mayor puntuación, mayor identificación con la variable y mejor calidad de vida. ${ }^{\circ}$ A mayor puntuación, peor calidad de vida. * $p<0.05 * * p<0.01$

Fuente: elaboración propia. 
De otro lado, tampoco se hallaron discrepancias significativas al analizar por separado la puntuación otorgada a cada una de las zonas del cuerpo $(p>0.05)$, ni en la puntuación media obtenida de dichos valores $(p>0.05)$. No obstante, sí se encontraron diferencias en la valoración del pecho/ tórax, que resultó significativamente mayor en las pacientes reconstruidas $(p<0.01)$. Asimismo, las pacientes mastectomizadas mostraron un nivel de insatisfacción significativamente mayor con dicha zona corporal $(p<0.01)$, al compararlas con las mujeres reconstruidas (Tabla 2 ).

$\mathrm{Al}$ comprobar las diferencias en las variables estudiadas según los cuatro subgrupos quirúrgicos establecidos (mastectomía simple unilateral, mastectomía radical modificada unilateral, reconstrucción mamaria inmediata y reconstrucción mamaria diferida), no se hallaron diferencias significativas entre los dos subgrupos de pacientes mastectomizadas con respecto al nivel de satisfacción con el resultado estético de la intervención quirúrgica ( $p$ $>0.05$ ). Tampoco discreparon de manera significativa en relación al nivel de neuroticismo ( $p>0.05)$, al nivel de autoestima ( $p>0.05)$, ni en ninguna de las variables que determinan el grado de satisfacción corporal $(p>0.05)$. Por el contrario, las pacientes con mastectomía simple sí mostraron un nivel de funcionamiento sexual significativamente superior al de las mujeres con mastectomía radical modificada $(p<0.05)$, así como una menor sintomatología en el brazo $(p<0.05)$ secundaria a la intervención quirúrgica. No se detectaron diferencias significativas entre las pacientes, según el tipo de reconstrucción mamaria (inmediata o diferida)

TABLA 2

Comparación entre pacientes mastectomizadas y reconstruidas en el grado de satisfacción corporal

\begin{tabular}{|c|c|c|c|c|}
\hline \multirow[b]{2}{*}{ Variables } & \multicolumn{2}{|c|}{ Tipo de cirugía } & \multirow[t]{2}{*}{$\begin{array}{c}\text { Estadístico de } \\
\text { contraste }\end{array}$} & \multirow[t]{2}{*}{$p$} \\
\hline & $\begin{array}{c}\text { Mastectomía } \\
(N=36) \\
M(D E)\end{array}$ & $\begin{array}{c}\text { Reconstrucción } \\
(N=36) \\
M(D E)\end{array}$ & & \\
\hline \multicolumn{5}{|l|}{ Satisfacción corporal } \\
\hline Cabello & $6.25(2.02)$ & $6.72(2.03)$ & $t(70)=-0.98$ & 0.327 \\
\hline Cutis & $6.5(1.97)$ & $6.78(2.01)$ & $t(70)=-0.59$ & 0.557 \\
\hline Ojos & $6.89(1.61)$ & $7.06(2.12)$ & $\mathrm{t}(70)=-0.375$ & 0.709 \\
\hline Nariz & $6.25(1.9)$ & $6.31(2.47)$ & $t(70)=-0.106$ & 0.916 \\
\hline Boca & $6.53(1.96)$ & $6.69(1.81)$ & $\mathrm{U}=639.5$ & 0.922 \\
\hline Labios & $6.75(1.88)$ & $6.69(1.95)$ & $\mathrm{t}(70)=0.123$ & 0.903 \\
\hline Cuello & $6.36(2.19)$ & $6.78(1.74)$ & $t(70)=-0.893$ & 0.375 \\
\hline Pecho/Tórax & $1.33(1.78)$ & $5.31(3.31)$ & $\mathrm{U}=218.5$ & $0.000 * *$ \\
\hline Brazos & $6.26(1.97)$ & $6.67(1.82)$ & $\mathrm{t}(70)=-0.683$ & 0.497 \\
\hline Manos & $6.31(2.01)$ & $6.56(1.55)$ & $t(70)=-0.590$ & 0.557 \\
\hline Abdomen & $4.94(2.52)$ & $5.64(2.4)$ & $t(70)=-1.19$ & 0.236 \\
\hline Cintura & $5.69(2.37)$ & $5.70(2.39)$ & $\mathrm{t}(70)=-0.148$ & 0.883 \\
\hline Genitales & $6.44(2.17)$ & $6.81(1.7)$ & $\mathrm{t}(70)=-0.785$ & 0.435 \\
\hline Nalgas & $5.58(2.54)$ & $6.47(1.76)$ & $t(70)=-1.72$ & 0.089 \\
\hline Caderas & $5.86(2.52)$ & $6.36(1.88)$ & $\mathrm{t}(70)=-0.953$ & 0.344 \\
\hline Muslos & $5.53(2.74)$ & $6.36(1.77)$ & $t(59.96)=-10.53$ & 0.131 \\
\hline Piernas & $5.75(2.72)$ & $6.42(2.11)$ & $t(70)=-1.15$ & 0.251 \\
\hline Piel & $6.86(1.6)$ & $6.67(1.8)$ & $\mathrm{t}(70)=0.483$ & 0.631 \\
\hline Puntuación media distintas zonas del cuerpo & $5.89(1.75)$ & $6.44(1.43)$ & $\mathrm{t}(70)=-1.44$ & 0.152 \\
\hline
\end{tabular}

Nota: $N=72$. A mayor puntuación, mayor identificación con la variable. $* * p<0.01$

Fuente: elaboración propia. 
TABLA 3

Correlación entre satisfacción con el resultado estético, autoestima e imagen corporal

\begin{tabular}{lcc}
\hline \multicolumn{1}{c}{ Correlaciones entre variables } & $r$ & $p$ \\
\hline Satisfacción resultado estético - Satisfacción con pecho/tórax & 0.671 & $0.000^{* *}$ \\
Autoestima - Satisfacción resultado estético & 0.314 & $0.007^{* *}$ \\
Autoestima - Neuroticismo & -0.362 & $0.002^{* *}$ \\
Autoestima - Imagen Corporal ${ }^{\circ}$ & -0.466 & $0.000^{* *}$ \\
Autoestima - Satisfacción con pecho/tórax $_{\text {Autoestima - Puntuación media satisfacción corporal }}$ & 0.341 & $0.003^{* *}$ \\
Imagen Corporal $^{\circ}$ - Satisfacción resultado estético & 0.338 & $0.004^{* *}$ \\
Imagen Corporal $^{\circ}$ - Satisfacción con pecho/tórax & -0.619 & $0.000^{* *}$ \\
\hline
\end{tabular}

Nota. $N=72 .{ }^{\circ}$ A mayor puntuación, peor calidad de vida. $* * p<0.01$

Fuente: elaboración propia.

en ninguna de las variables estudiadas $(p>0.05)$, a excepción del nivel de autoestima que fue hallado significativamente superior en las mujeres reconstruidas de manera inmediata $(p<0.05)$.

En relación con el segundo objetivo de este estudio, se quisieron establecer las relaciones existentes entre las principales variables psicológicas en las que se hallaron discrepancias significativas entre las pacientes mastectomizadas y reconstruidas (satisfacción con el resultado estético, autoestima, imagen corporal y satisfacción con la zona corporal del pecho/tórax). Para ello, se utilizó el coeficiente de correlación de Pearson (Tabla 3).

Para comprobar el tercer y último objetivo propuesto, se empleó el coeficiente de correlación de Pearson para cada uno de los dos grupos muestrales.

Se comprobó que el deterioro en la imagen corporal manifestado por las pacientes mastectomizadas, se modificaba en función del tiempo transcurrido desde que recibieron el diagnóstico oncológico $(p<0.05)$, así como del tiempo transcurrido desde la última cirugía $(p<0.01)$. Lo mismo ocurrió con el disfrute sexual $(p<0.05)$, así como con la gran mayoría de las escalas que evaluaban la calidad de vida específica en cáncer de mama $(p<0.01)$. Del mismo modo, el nivel de autoestima de estas pacientes se relacionó con el tiempo transcurrido desde que se les practicó la mastectomía $(p<0.05)$ (Tablas 4 y 5).

En el grupo de las pacientes reconstruidas se apreció que el tiempo acontecido desde el diagnóstico oncológico estuvo relacionado tan sólo con el disfrute sexual $(p<0.05)$. Igualmente, el nivel de satisfacción con el resultado estético de la intervención quirúrgica, el grado de preocupación por el futuro, así como la sintomatología secundaria al tratamiento quirúrgico y radioterápico, se hallaron relacionadas con el tiempo transcurrido desde que se practicó la última cirugía mamaria a la paciente $(p<0.05)$ (Tablas 4 y 5$)$.

\section{TABLA 4}

Correlación entre las variables psicológicas y el tiempo transcurrido desde el diagnóstico oncológico en las pacientes mastectomizadas y reconstruidas

\begin{tabular}{lcc}
\hline \multicolumn{1}{c}{ Correlaciones entre variables } & $r$ & $p$ \\
\hline Mastectomizadas (N=36) & & \\
\hline Satisfacción estética - Tiempo desde el diagnóstico & -0.047 & 0.787 \\
Neuroticismo - Tiempo desde el diagnóstico & -0.026 & 0.879 \\
Autoestima - Tiempo desde el diagnóstico & 0.327 & 0.052 \\
Imagen Corporal ${ }^{\circ}$ - Tiempo desde el diagnóstico & -0.396 & $0.017^{*}$ \\
Disfrute del Sexo a - Tiempo desde el diagnóstico & 0.397 & $0.016^{*}$ \\
\hline
\end{tabular}




\begin{tabular}{|c|c|c|}
\hline Correlaciones entre variables & $r$ & $p$ \\
\hline Preocupación por el futuro $^{\circ}$ - Tiempo desde el diagnóstico & -0.668 & $0.000 * *$ \\
\hline Síntomas del brazo por la cirugía ${ }^{\circ}$ - Tiempo desde el diagnóstico & -0.501 & $0.002 * *$ \\
\hline Síntomas de la mama por la Radioterapia ${ }^{\circ}$ - Tiempo desde el diagnóstico & -0.506 & $0.002 * *$ \\
\hline Síntomas secundarios por la Quimioterapia ${ }^{\circ}$ - Tiempo desde el diagnóstico & -0.435 & $0.008^{* *}$ \\
\hline Síntomas secundarios por la Hormonoterapia ${ }^{\circ}$ - Tiempo desde el diagnóstico & -0.64 & $0.000 * *$ \\
\hline Preocupación por la caída del cabello ${ }^{\circ}$ - Tiempo desde el diagnóstico & -0.435 & $0.008 * *$ \\
\hline \multicolumn{3}{|l|}{ Reconstruidas $(\mathrm{N}=36)$} \\
\hline Satisfacción estética - Tiempo desde el diagnóstico & 0.143 & 0.407 \\
\hline Neuroticismo - Tiempo desde el diagnóstico & -0.067 & 0.697 \\
\hline Autoestima - Tiempo desde el diagnóstico & -0.293 & 0.083 \\
\hline Disfrute del Sexo ${ }^{\text {a }}$ - Tiempo desde el diagnóstico & 0.359 & $0.031 *$ \\
\hline
\end{tabular}

Nota. $N=72 .{ }^{a}$ A mayor puntuación, mayor identificación con la variable y mejor calidad de vida. ${ }^{\circ}$ A mayor puntuación, peor calidad de vida. * $p<0.05 * * p<0.01$

Fuente: elaboración propia.

\section{TABLA 5}

Correlación entre las variables psicológicas y el tiempo transcurrido desde la realización de la última cirugía en las pacientes mastectomizadas y reconstruidas

\begin{tabular}{|c|c|c|}
\hline Correlaciones entre variables & $r$ & $p$ \\
\hline \multicolumn{3}{|l|}{ Mastectomizadas $(N=36)$} \\
\hline Satisfacción estética - Tiempo desde la última cirugía & -0.066 & 0.7 \\
\hline Neuroticismo - Tiempo desde la última cirugía & -0.031 & 0.858 \\
\hline Autoestima - Tiempo desde la última cirugía & 0.334 & $0.047 *$ \\
\hline Imagen Corporal ${ }^{\circ}$ - Tiempo desde la última cirugía & -0.428 & $0.009 * *$ \\
\hline Disfrute del Sexo ${ }^{a}$ - Tiempo desde la última cirugía & 0.373 & $0.025^{*}$ \\
\hline Preocupación por el futuro ${ }^{\circ}$ - Tiempo desde la última cirugía & -0.683 & $0.000 * *$ \\
\hline Síntomas del brazo por la cirugía ${ }^{\circ}$ - Tiempo desde la última cirugía & -0.506 & $0.002 * *$ \\
\hline Síntomas de la mama por la Radioterapia ${ }^{\circ}$ - Tiempo desde la última cirugía & -0.509 & $0.002 * *$ \\
\hline Síntomas secundarios por la Quimioterapia ${ }^{\circ}$ - Tiempo desde la última cirugía & -0.438 & $0.008 * *$ \\
\hline Síntomas secundarios por la Hormonoterapia ${ }^{\circ}$ - Tiempo desde la última cirugía & -0.647 & $0.000 * *$ \\
\hline Preocupación por la pérdida del pelo ${ }^{\circ}$ - Tiempo desde la última cirugía & -0.427 & $0.009 * *$ \\
\hline \multicolumn{3}{|l|}{ Reconstruidas $(\mathrm{N}=36)$} \\
\hline Satisfacción estética - Tiempo desde la última cirugía & 0.359 & $0.032 *$ \\
\hline Neuroticismo - Tiempo desde la última cirugía & -0.201 & 0.239 \\
\hline Autoestima - Tiempo desde la última cirugía & 0.212 & 0.215 \\
\hline Preocupación por el futuro ${ }^{\circ}$ - Tiempo desde la última cirugía & -0.337 & $0.045^{*}$ \\
\hline Síntomas del brazo por la cirugía ${ }^{\circ}$ - Tiempo desde la última cirugía & -0.346 & $0.039 *$ \\
\hline Síntomas de la mama por la Radioterapia ${ }^{\circ}$ - Tiempo desde la última cirugía & -0.372 & $0.025^{*}$ \\
\hline
\end{tabular}

Nota. $N=72$ a $^{\text {a }}$ mayor puntuación, mayor identificación con la variable y mejor calidad de vida. ${ }^{\circ}$ A mayor puntuación, peor calidad de vida. $* p<0.05 * * p<0.01$

Fuente: elaboración propia. 


\section{Discusión}

Las mujeres con reconstrucción mamaria manifestaron una mayor satisfacción con el resultado estético de la intervención, tal y como se había señalado previamente (Alderman et al., 2000; Cocquyt et al., 2003; Contant et al., 2000; Janni et al., 2001; Nicholson et al., 2007), así como un mayor nivel de autoestima, coincidiendo con los resultados obtenidos por otros autores (Al-Ghazal, Sully, Fallowfield \& Blamey, 2000).

El nivel de neuroticismo se distribuyó de manera homogénea en los dos grupos muestrales, en oposición a los hallazgos de Keith et al. (2003) que afirmaban un menor deseo de someterse a reconstrucción mamaria cuanto mayor nivel de neuroticismo manifestasen las pacientes.

Al estudiar la calidad de vida específica en pacientes con cáncer de mama, se comprobó que las mujeres reconstruidas mostraban una mejor imagen corporal, junto a una menor preocupación por el futuro o por la pérdida del cabello. Igualmente, describieron una menor sintomatología experimentada en el brazo, en la mama, o secundaria a la quimioterapia y hormonoterapia. No en vano, la mayoría de los estudios coinciden en afirmar que aquellas mujeres que son sometidas a reconstrucción mamaria postmastectomía, experimentan significativas mejorías en su imagen corporal y autoestima frente a las pacientes que no son reconstruidas (Al-Ghazal, Fallowfield et al., 2000).

Por el contrario, las pacientes mastectomizadas y reconstruidas no discrepaban en relación al nivel de funcionamiento sexual o de disfrute del sexo, situándose en la línea de lo mencionado por Schover et al. (1995).

Analizando el grado de satisfacción corporal de cada mujer, no se detectaron diferencias entre las pacientes mastectomizadas y reconstruidas, con respecto a la valoración de cada zona de su cuerpo por separado, a excepción del pecho/tórax. Dicha zona corporal fue peor valorada por las pacientes mastectomizadas, siendo además identificada como una parte problemática de su anatomía. Por tanto, se puede afirmar que la menor satisfacción con la imagen corporal que revelaron las pacien- tes mastectomizadas, se circunscribe tan solo a la insatisfacción que expresaron con el aspecto de su pecho/tórax.

De otro lado y según los resultados obtenidos, se puede concluir que las únicas diferencias halladas entre las pacientes según el tipo de mastectomía realizada, se basó en un mayor funcionamiento sexual en las pacientes con mastectomía simple, así como una menor sintomatología secundaria a la cirugía. Ello se debe a que la mastectomía radical modificada implica, además de la extirpación de toda la glándula mamaria (mastectomía simple), el vaciamiento axilar completo (Del Val, 2006). Por ello, estas pacientes aquejan una mayor sintomatología en el brazo secundaria a la cirugía. Estas molestias podrían explicar el menor apetito sexual de estas mujeres, traducido en un peor funcionamiento sexual.

Al determinar las discrepancias existentes entre las pacientes con reconstrucción mamaria, según el momento temporal en el que dicha intervención hubiera sido llevada a cabo (de manera inmediata o diferida), se comprobó la ausencia de diferencias significativas en la mayoría de las variables psicológicas estudiadas, siguiendo la misma línea de lo mencionado previamente por diversos autores (Harcourt et al., 2003; Nicholson et al., 2007; Nissen et al., 2001). Tan solo se halló un mayor nivel de autoestima en las pacientes con reconstrucción mamaria inmediata, frente a las mujeres con reconstrucción diferida, tal y como señalaron otros estudios (Al-Ghazal, Sully et al., 2000). Estas discrepancias podrían deberse al hecho de que una reconstrucción mamaria temprana reduce los efectos psicológicos negativos producidos por la extirpación de la mama (Schain, 1991). Según esto, la reconstrucción mamaria se consideraría el proceso de restitución de la imagen corporal y del bienestar psicológico de las pacientes mastectomizadas (Marín-Gutzke \& Sánchez-Olaso, 2010).

No obstante, y a pesar de que los resultados indican una mayor autoestima, calidad de vida y satisfacción corporal con la zona del pecho/tórax en las pacientes con reconstrucción mamaria, es plausible contemplar hipótesis causales alternativas a las anteriormente planteadas. De este modo, la 
investigación ha delimitado algunas de las características sociodemográficas más comunes en las mujeres que eligen reconstruirse la mama. Estas pacientes han sido descritas como mujeres más jóvenes, de mayor nivel educativo (Morrow et al., 2005; Rowland et al., 2000), con pareja estable (Rowland et al., 2000), y con etapas menores de evolución del cáncer (Morrow et al., 2005). Estas características podrían delimitar el perfil de una mujer con mayores recursos sociales y personales, los cuales les permiten desplegar estrategias de afrontamiento diferenciales ante la amputación mamaria. Por ello, gozarían de una menor alteración de su imagen corporal, así como de un mayor nivel de autoestima. Ello, se situaría en la misma línea que lo señalado anteriormente por otros autores, al mencionar las estrategias de afrontamiento empleadas por las pacientes como uno de los factores determinantes del ajuste psicológico a la enfermedad (Anarte et al., 2001; Cardenal et al., 2008; Collie et al., 2005; Font \& Cardoso, 2009; Neipp et al., 2009). Asimismo, y según afirmó Raich (2000), la menor alteración de la imagen corporal de las pacientes reconstruidas podría estar mediada por el significado que estas mujeres le atribuyen en función de unos factores sociales y culturales diferentes a los que puedan rodear a las mujeres mastectomizadas, en estrecha relación con sus habilidades de afrontamiento y características de personalidad.

Una vez obtenidas todas las discrepancias en las variables objeto de estudio, según el tipo de cirugía practicada a la paciente, se comprobó si estas variables se relacionaban entre sí. De este modo, los resultados del estudio correlacional señalaron que aquellas pacientes con mayor autoestima, expresaron también una mayor satisfacción con el resultado estético de la intervención quirúrgica, así como una mayor satisfacción con su cuerpo y concretamente con la zona corporal del pecho/tórax. Asimismo, mostraron un menor nivel de neuroticismo tal y como ha sido señalado previamente (Buunk et al., 1990), junto a un menor deterioro de su imagen corporal.

De otro lado, en el grupo de las pacientes mastectomizadas se comprobó que cuanto mayor número de meses hubieran sucedido desde que recibieron el diagnóstico oncológico así como desde que se les practicó la mastectomía, expresaron una mejoría en su imagen corporal y disfrute sexual, así como una menor preocupación por el futuro o por la pérdida de cabello, unido a una reducción de la sintomatología secundaria a los tratamientos médicos. De este modo, un mayor tiempo transcurrido desde el diagnóstico oncológico se relacionó con una mejor calidad de vida (Dorval, Mounsell, Deschenes, Brisson \& Masse, 1998). Asimismo, un menor nivel de autoestima de estas pacientes se asoció a un menor tiempo transcurrido desde la mastectomía. Resultados similares han sido descritos por otros autores, al afirmar que el transcurso del tiempo desde la mastectomía mejora la imagen corporal y el bienestar físico y funcional de estas mujeres (Arora et al., 2001).

Por el contrario, el grado de satisfacción con el resultado estético de la mastectomía practicada a estas pacientes no dependió del tiempo que hubiese transcurrido desde dicha intervención quirúrgica, ni tampoco desde su diagnóstico. Según estos hallazgos, se podría concluir que, si bien el paso del tiempo mejora el deterioro de la imagen corporal que padecen las pacientes a consecuencia de la amputación mamaria, este no influye en su insatisfacción con el resultado estético consecuente a la mastectomía.

En relación con las pacientes con reconstrucción mamaria, un mayor tiempo transcurrido desde que recibieron el diagnóstico oncológico se relacionó tan solo con un mayor disfrute sexual. Cabe destacar que, a diferencia de los resultados obtenidos en las mujeres mastectomizadas, las pacientes reconstruidas sí mostraron una mayor satisfacción con el resultado estético de la intervención quirúrgica, a medida que hubiese transcurrido más tiempo desde que se les practicó la reconstrucción mamaria. Asimismo, en estas pacientes, el grado de preocupación por el futuro así como la sintomatología padecida en el brazo a consecuencia de la cirugía o en la mama secundaria a la radioterapia, se redujeron a medida que hubiese transcurrido un mayor número de meses desde que fueron reconstruidas.

Según los datos expuestos anteriormente, se deben considerar algunos aspectos relevantes. Es 
importante tener en cuenta que para las pacientes mastectomizadas (sea de manera simple o radical modificada) la "última cirugía practicada", constituye aquel acto quirúrgico en el cual se extirpa la mama a la paciente. Sin embargo, para la mujer con reconstrucción mamaria la variable última cirugía practicada, podía representar el primer o segundo acto quirúrgico para reconstruir la mama. Así pues, es lógico pensar que el mayor o menor tiempo transcurrido desde la última cirugía practicada a la paciente, no produzca los mismos efectos a nivel psicológico en las mujeres reconstruidas que en las mastectomizadas.

Una paciente reconstruida que acaba de ser sometida al primer o segundo acto quirúrgico de la reconstrucción, ya no está tan mutilada como cuando estaba mastectomizada, por ello es comprensible esperar que no se produzca un deterioro en relación a su imagen corporal o su autoestima (sí se producen en las mastectomizadas). Igualmente, una paciente mastectomizada que cuenta con menos tiempo transcurrido desde la última cirugía, tendrá también más reciente los tratamientos quimio y radioterápicos (estos se aplican tras la mastectomía), por esto es lógico que presente una mayor sintomatología secundaria a los tratamientos médicos y por tanto una menor calidad de vida. Asimismo, cuanto mayor tiempo haya transcurrido desde que la paciente fue sometida al último acto de la reconstrucción mamaria, manifestará una menor preocupación por el futuro de su enfermedad, así como una menor sintomatología secundaria al tratamiento quirúrgico y radioterápico. Ello es lógico si se tiene en cuenta que las pacientes reconstruidas de manera diferida han de esperar a completar los tratamientos adyuvantes, antes de someterse a la reconstrucción mamaria. Igual ocurre con las pacientes reconstruidas de forma inmediata, que por lo general tienen que finalizar los tratamientos médicos para poder pasar de la primera fase reconstructiva (colocación del expansor después de la extirpación de la mama), hasta la segunda fase (sustitución del expansor por la prótesis mamaria). De igual modo, el resultado estético de la reconstrucción mamaria mejora con el transcurso del tiempo debido, entre otros factores, al proceso de cicatrización, por lo que consecuentemente la satisfacción estética de estas pacientes también se incrementa a medida que acontecen un mayor número de meses.

En conclusión, a la luz de los resultados obtenidos se puede afirmar que el tiempo transcurrido desde la intervención quirúrgica y desde la comunicación del diagnóstico oncológico, constituyen variables altamente relevantes en la calidad de vida de las mujeres mastectomizadas. Asimismo, cabe destacar la relevancia que el nivel de autoestima de las pacientes representa en relación al nivel de satisfacción con el resultado quirúrgico, así como con el deterioro en la imagen corporal. De este modo, las intervenciones psico-oncológicas deben acontecer en los periodos pre y postquirúrgicos, con objeto de favorecer la adaptación de las pacientes a la cirugía, así como de combatir el descenso consecuente de su calidad de vida. Y teniendo en cuenta los resultados hallados y la contribución teórica de los mismos, sería recomendable considerar los siguientes aspectos, frente al abordaje de las pacientes con cáncer de mama: a) en primer lugar, aquellas mujeres mastectomizadas que tienen más reciente el diagnóstico oncológico, manifiestan una mayor preocupación por su futuro, así como una mayor sintomatología en el brazo, en la mama y secundaria al tratamiento hormonal, experimentando mayores dificultades en su imagen corporal, y menor disfrute en sus relaciones sexuales y b) en segundo lugar, las pacientes mastectomizadas que cuentan con menos tiempo transcurrido desde que se les practicó la mastectomía, ven mermado su nivel de autoestima.

Para finalizar, consideramos importante manifestar algunas limitaciones implicadas en este estudio, así como posibles propuestas de mejora para futuras investigaciones similares. En primer lugar, sería recomendable ampliar el número de pacientes evaluadas, ya que de esta forma se podrían concluir resultados mucho más generalizables. En segundo lugar, sería aconsejable estudiar las diferencias entre las pacientes mastectomizadas y reconstruidas, de manera longitudinal. Así, se realizaría una evaluación psicológica antes y después de que fueran sometidas a la mastectomía, llevando a cabo nuevas mediciones cuando un número 
considerable de estas mujeres hubieran llevado a cabo la reconstrucción mamaria. De este modo, se estudiaría la evolución temporal de la sintomatología psicológica, junto a la influencia que el tipo de cirugía mamaria pudiera causar en la misma, y se contaría con una medida premórbida. Igualmente, todas las pacientes contarían con el mismo intervalo temporal transcurrido desde el diagnóstico y desde la mastectomía previa. Por último, se debe tener en cuenta el empleo de un grupo control, conformado por pacientes con cirugía conservadora, para realizar comparaciones con otro tipo de cirugías mamarias.

\section{Referencias}

Alderman, A. K., Wilkins, E. G., Lowery, J. C., Kim, M. \& Davis, J. A. (2000). Determinants of patient satisfaction in postmastectomy breast reconstruction. Plastic and Reconstructive Surgery, 106, 769-776.

Al-Ghazal, S. K., Fallowfield, L. \& Blamey, R. W. (2000). Comparison of psychological aspects and patient satisfaction following breast conserving surgery, simple mastectomy and breast reconstruction. European Journal of Cancer, 36, 1938-1943.

Al-Ghazal, S. K., Sully, L., Fallowfield, L. \& Blamey, R. W. (2000). The psychological impact of immediate rather than delayed breast reconstruction. European Journal of Surgical Oncology, 26, 17-19.

Anarte, M. T., Esteve, R., Ramírez, C., López, A. E. \& Amorós, F. J. (2001). Adaptación al castellano de la escala Courtauld de Control Emocional en Pacientes con Cáncer. Psicothema, 13, 636 -642.

Arora, N. K., Gustafson, D. H., Hawkins, R. P., Mctavish, F., Cella, D. F., Pingree, S. et al. (2001). Impact of surgery and chemotherapy on the quality of life of younger women with breast carcinoma. A prospective study. Cancer, 92, 1288-1298.

Arraras, J. I., Tejedor, M., Illaramendi, J. J., Vera, R., Pruja, E., Marcos, M. et al. (2001). El Cuestionario de Calidad de Vida para Cáncer de Mama de la EORTC, QLQ-BR23: estudio psicométrico con una muestra española. Psicología Conductual, 9, 81-97.

Atisha, D., Alderman, A. K., Lowery, J. C., Kuhn, L. E., Davis, J. \& Wilkins, E. G. (2008). Prospec- tive analysis of long-term psychosocial outcomes in breast reconstruction. Two-year postoperative results from the Michigan Breast Reconstruction Outcomes Study. Annals of Surgery, 247, 1019-1028.

Avis, N., Crawford, S. \& Manuel, J. (2004). Psychosocial problems among younger women with breast cancer. Psycho-Oncology, 13, 295-308.

Buick, D., Petrie, K., Booth, R., Probert, J., Benjamin, C. \& Harvey, V. (2000). Emotional and functional impact of radiotherapy and chemotherapy on patients with primary breast cancer. Journal of Psychosocial Oncology, 18, 39-62.

Buunk, B. P., Collins, R. L., Taylor, S. E., VanYperen, N. W. \& Dakof, G. A. (1990). The affective consequences of social comparison: Either direction has its ups and downs. Journal of Personality and Social Psychology, 59, 1238-1249.

Cardenal, V., Ortiz-Tallo, M., Martín-Frias, I. \& Martínez-Lozano, J. (2008). Estresores vitales, evitación emocional y cáncer de mama. Spanish Journal of Psychology, 11, 522-530.

Cocquyt, V. F., Blondeel, P. N., Depypere, H. T., Karlien, A., Van De Sijpe, K. A., Daems, K. K. et al. (2003). Better cosmetic results and comparable quality of life after skin-sparing mastectomy and immediate autologous breast reconstruction compared to breast conservative treatment. British Journal of Plastic Surgery, 56, 462-470.

Collie, K., Wong, P., Tilston, J., Butler, L. D., TurnerCobb, J., Kreshka, M. A. et al. (2005). Self-efficacy, coping, and difficulties interacting with health care professionals among women living with breast cancer in rural communities. Psycho-Oncology, 14, 901-912.

Contant, C. M., Van Wersch, A. M., Wiggers, T., Tjong, R. \& Van Geel, A. N. (2000). Motivations, satisfaction, and information of immediate breast reconstruction following mastectomy. Patient Education and Counseling, 40, 201-208.

Cordova, M. J., Cunninggham, L. L., Carlson, C. R. \& Andrykowski, M. A. (2001). Social constraints, cognitive processing and adjustment to breast cancer. Journal of Consulting and Clinical Psychology, 69, 706-711. 
Costa, G. \& Gil, F. (2008). Respuesta cognitiva y crecimiento postraumático durante el primer año del diagnóstico de cáncer. Psicooncología, 5, 27-37.

Curbow, B. \& Somerfield, M. (1991). Use of the Rosenberg Self-Esteem Scale with adult cancer patients. Journal of Psychosocial Oncology, 9, 113-131.

Del Val, J. M. (2006). Mastectomías: indicaciones y aspectos técnicos. En A. Sierra, A. Piñero \& J. Illana (Eds.), Cirugía de la mama (pp. 367-373). Madrid: Aran Ediciones.

Dorval, M., Mounsell, E., Deschenes, L., Brisson, J. \& Masse, B. (1998). Long-term quality of life after breast cancer: Comparison of 8-year survivors with population controls. Journal of Clinical Oncology, $16,487-494$.

Elder, E. E., Brandberg, Y., Bjorklund, T., Rylanderc, R., Lagergren, J., Jurell, G. et al. (2005). Quality of life and patient satisfaction in breast cancer patients after immediate breast reconstruction: A prospective study. Breast, 14, 201-208.

Fernández, A. I. (2004). Alteraciones psicológicas asociadas a los cambios en la apariencia física en pacientes oncológicos. Psicooncología, 1, 169-180.

Ferrell, B. R., Grant, M., Funk, B., Otis-Green, S. \& Garcia, N. (1997). Quality of life in breast cancer: Part I: Physical and social well-being. Cancer Nursing, 20, 398-408.

Font, T. \& Cardoso, A. (2009). Afrontamiento en cáncer de mama: pensamientos, conductas y reacciones emocionales. Psicooncología, 6, 27-42.

Francis, L. J., Brown, L. B. \& Philipehalk, R. (1992). The development of an abbreviated form of the revised Eysenck Personality Questionnaire (EPQR-A): Its use among students in England, Canada, the USA and Australia. Personality and Individual Differences, 13, 443-449.

Ganz, P. A. (2008). Psychological and social aspects of breast cancer. Oncology, 22, 642-653.

García-Tornel, S. \& Gaspá, J. (1997). Control de Salud de la población adolescente. Pediatría Integral, 2, 285-295.

Harcourt, D. M., Rumsey, N. J., Ambler, N. R., Cawthorn, S. J., Reid, C. D., Maddox, P. R. et al. (2003). The psychological effect of mastectomy with or without breast reconstruction: A prospective, mul- ticenter study. Plastic and Reconstructive Surgery, 111, 1060-1068.

Hopwood, P., Fletcher, I., Lee, A. \& Al-Ghazal, S. (2001). A body image scale for use with cancer patients. European Journal of Cancer, 37, 189-197.

Hopwood, P. \& Maguire, G. P. (1998). Body image problems in cancer patients. The British Journal of Psychiatry, 153, 47-50.

Janni, W., Rjosk, D., Dimpfl, T., Haertl, K., Strobl, B., Hepp, F. et al. (2001). Quality of life influenced by primary surgical treatment for stage I-III breast cancer long-term follow up of a matched pair analysis. Annals of Surgical Oncology, 8, 542-548.

Keith, D. J., Walker, M. B., Walker, M. B., Walker, L. G., Heys, S. D., Sarkar, T. K. et al. (2003). Women who wish breast reconstruction: Characteristics, fears, and hopes. Plastic and Reconstructive Surgery, 111, 1051-1159.

Lechner, S. C., Zakowski, S. G., Antoni, M. H., Greenhawt, M. \& Block, P. (2003). Do sociodemographic and disease related variables influence benefitfinding in cancer patients? Psycho-Oncology, 12, 491-499.

Manne, S., Ostrff, J., Winkel, G., Goldstein, L., Fox, K. \& Grana, G. (2004). Posttraumatic growth after breast cancer: Patient, partner and couple perspectives. Psychosomatic Medicine, 66, 442-454.

Manos, D., Sebastián, J., Bueno, M. J., Mateos, N. \& De la Torre, A. (2005). Body image in relation to selfesteem in a sample of Spanish women with earlystage breast cancer. Psicooncología, 2, 103-116.

Marín-Gutzke, M. \& Sánchez-Olaso, A. (2010). Reconstructive surgery in young women with breast cancer. Breast Cancer Research and Treatment, 123, 67-74.

Millar, K., Purushotham, A. D., McLatchie, E., George, W. D. \& Murray, G. D. (2005). A 1-year prospective study of individual variation in distress, and illness perceptions, after treatment for breast cancer. Journal of Psychosomatic Research, 58, 335-342.

Min, S. Y., Kim, H. Y., Jung, S., Kwon, Y., Shin, K. H., Lee, S. et al. (2010). Oncological safety and quality of life associated with mastectomy and immediate breast reconstruction with a latissimus dorsi myocutaneous flap. The Breast Journal, 16, 356-361. 
Miró, M. A. (2006). Avaluació de la imatge corporal per a la detecció precoç dels trastorns alimentaris. Tesis de Doctorado para la obtención del título de Doctora en Psicología, Facultad de Psicología, Universidad Autónoma de Barcelona, Barcelona, España.

Morrow, M., Mujahid, M., Lantz, P. M., Janz, N. K., Fagerlin, A., Schwartz, K. et al. (2005). Correlatos of breast reconstruction: Results from a population-based study. Cancer, 104, 2340-2346.

Mose, S., Budischewski, K. M., Rahn, A. N., ZanderHeinz, A. C., Bormeth, S. \& Bottcher, H. D. (2001). Influence of irradiation on therapy-associated psychological distress in breast carcinoma patients. International Journal of Radiation Oncology, Biology, Physics, 51, 1328-1335.

Muñoz, A. (2010). Quality of life during treatment in young women with breast cancer. Breast Cancer Research and Treatment, 123, 75-77.

Neipp, M. C., López-Roig, S., Terol, M. C. \& Pastor, M. A. (2009). Changes in control beliefs, emotional status and psychosocial adaptation among women with breast cancer. Anales de Psicología, 25, 36-43.

Nicholson, R. M., Leinster, S. \& Sassoon, E. M. (2007).

A comparison of the cosmetic and psychological outcome of breast reconstruction, breast conserving surgery and mastectomy without reconstruction. The Breast, 16, 396-410.

Nissen, M. J., Swenson, K. K., Ritz, L. J., Farrell, J. B., Sladek, M. L. \& Lally, R. M. (2001). Quality of life after breast carcinoma surgery: A comparison of three surgical procedures. Cancer, 91, 1238-1246.

Quintard, B. \& Lakdja, F. (2008). Assessing the effect of beauty treatments on psychological distress, body image, and coping: A longitudinal study of patients undergoing surgical procedures for breast cancer. Psycho-Oncology, 17, 1032-1038.

Raich, R. M. (2000). Trastornos de la imagen corporal. En R. M. Raich (Ed.), Imagen corporal. Conocer y valorar el propio cuerpo (pp. 27-64). Madrid: Ediciones Pirámide.

Raich, R., Torras, J. \& Sánchez-Carracedo, D. (2001, septiembre). Body image in a sample of adolescent students. Book of Abstracts of the VII European Council on Eating Disorders, Barcelona, 15.

Rosenberg, M. (1965). Society and the adolescent child. Princeton, NJ: Princeton University Press.
Rosenberg, M. (1986). Self-concept from middle childhood through adolescence. En J. Suls \& A. G. Greenwald (Eds.), Psychological perspectives on the self (pp. 107-135). Hillsdale, NJ: Erlbaum.

Rowland, J. H., Desmond, K. A., Meyerowitz, B. E., Belin, T. R., Wyatt, G. E. \& Ganz, P. A. (2000). Role of breast reconstructive surgery in physical and emotional outcomes among breast cancer survivors. Journal of the National Cancer Institute, 92, 1422-1429.

Rubino, C., Figus, A., Lorettu, L. \& Sechi, G. (2007). Post-mastectomy reconstruction: A comparative analysis on psychosocial and psychopathological outcomes. Journal of Plastic, Reconstructive $\mathcal{E}$ Aesthetic Surgery, 60, 509-518.

Salkind, N. J. (1999). Métodos de investigación. México: Prentice Hall.

Sandín, B., Valiente, R. M., Chorot, P., Olmedo, M. \& Santed, M .A. (2002). Versión española del cuestionario EPQR-Abreviado (EPQR-A) (I): análisis exploratorio de la estructura factorial. Revista de Psicopatología y Psicología Clínica, 7, 195-205.

Sandín, B., Valiente, R. M., Olmedo, M., Chorot, P. \& Santed, M. A. (2002). Versión española del cuestionario EPQR-Abreviado (EPQR-A) (II): replicación factorial, fiabilidad y validez. Revista de Psicopatología y Psicología Clínica, 7, 207-216.

Schain, W. S. (1991). Breast reconstruction. Update of psychosocial and pragmatic concerns. Cancer, 68, 1170-1175.

Schover, L. R., Yetman, R. J., Tauson, L. J., Meisler, E., Esselstyn, C. B., Hermann, R. E. et al. (1995). Partial mastectomy and breast reconstruction. A comparison of their effects on psychosocial adjustment, body image, and sexuality. Cancer, 75, 54-64.

Sellick, S. M. \& Edwardson, A. D. (2007). Screening new cancer patients for psychological distress using the hospital anxiety and depression scale. PsychoOncology, 16, 534-542.

Sprangers, M. A., Groenvold, M., Arraras, J. I., Franklin, J., Te Velde, A., Muller, M. et al. (1996). The European Organization for Research and Treatment of Cancer Breast Cancer-Specific Quality-of-Life Questionnaire Module: First results from a threecountry field study. Journal of Clinical Oncology, $14,2756-2768$. 
Stiegelis, H., Ranchor, A. \& Sanderman, R. (2004). Psychological functioning in cancer patients treated with radiotherapy. Patient Education and Counseling, 52, 131-141.

Torres, A. M. (2006). Relación entre el patrón de conducta Tipo C y el cáncer de mama. Universitas Psicologica, 5(3), 563-573.

Vázquez, A. J., Jiménez, R. \& Vázquez, R. (2004). Escala de Autoestima de Rosenberg: fiabilidad y validez en población clínica española. Apuntes de Psicología, 22, 247-255.

Vázquez, J. P., López, S. \& Martín, M. (2004). Linfedema. En C. Camps, J. Carulla, A. M. Casas, M.
González, J. Sanz-Ortiz \& V. Valentín (Eds.), Manual de Cuidados Continuos (pp. 379-390). Madrid: Sociedad Española de Oncología Médica, Sección de Cuidados Continuos.

Whelan, T. J., Levine, M., Julian, J., Kirkbride, P. \& Skingley, P. (2000). The effects of radiation therapy on quality of life of women with breast carcinoma: Results of a randomized trial (Ontario Clinical Oncology Group). Cancer, 88, 2260-2266.

White, C. A. (2000). Body image dimensions and cancer: A heuristic cognitive behavioural model. Psycho-Oncology, 9, 183-192. 
